\title{
Current Treatment of Cryoglobulinemic Vasculitis
}

Sarah Goglin, MD

Sharon A. Chung, MD, MAS*

\author{
Address \\ ${ }^{*}$ Rosalind Russell/Ephraim P. Engleman Rheumatology Research Center, University \\ of California, San Francisco, San Francisco, CA, 94143, USA \\ Email: sharon.chung@ucsf.edu
}

Published online: 29 April 2016

(C) Springer International Publishing AG 2016

This article is part of the Topical Collection on Vasculitis

Keywords Cryoglobulinemic vasculitis - Hepatitis C · Interferon - Antiviral therapy · Rituximab · Plasma exchange

\section{Opinion statement}

For patients with cryoglobulinemic vasculitis, we consider treatment of the underlying disorder (if present) to be first-line therapy. For hepatitis C virus (HCV)-associated cryoglobulinemic vasculitis, antiviral therapy directed at HCV should be considered firstline treatment for patients who are candidates. Interferon-free direct acting antiviral regimens have been shown to be very effective in the treatment of chronic HCV infection and preliminary results of the use of these regimens are promising for the treatment of $\mathrm{HCV}$-associated cryoglobulinemic vasculitis. Close collaboration with a gastroenterologist or hepatologist is needed, given the complexity in choosing from the various treatment options. Patients with severe life- or organ-threatening manifestations of cryoglobulinemic vasculitis may benefit by treatment with rituximab in addition and potentially prior to the initiation of antiviral therapy. We also recommend treatment with rituximab in patients who have a contraindication to or who have failed antivirals. Plasmapheresis, in conjunction with immunosuppression, can be used as adjunctive therapy to antiviral and/or rituximab therapy for patients with severe organ- or lifethreatening disease. Cyclophosphamide use is best reserved for patients with severe disease who are unable to be treated with antiviral or rituximab therapy. The role of glucocorticoid use remains poorly defined, with some studies supporting moderate or high-dose prednisone use to treat active disease.

\section{Introduction}

Cryoglobulins are circulating immunoglobulins that precipitate in vitro at temperatures below 37 degrees Celsius and dissolve with rewarming. They are usually classified into one of three categories [1]. Type I cryoglobulins are isolated monoclonal immunoglobulins, which are usually
IgG or IgM, and are found in patients with lymphoproliferative disorders, such as Waldenstrom's macroglobulinemia, multiple myeloma and monoclonal gammopathy of unknown significance (MGUS). Type II cryoglobulins are a mixture of polyclonal immunoglobulins in association 
with a monoclonal immunoglobulin, usually IgM or IgA, with rheumatoid activity. Type III cryoglobulins are polyclonal immunoglobulins without a monoclonal component. Type II and type III cryoglobulins are referred to as mixed cryoglobulins because they are composed of both IgG and IgM.

The clinical syndrome associated with type I cryoglobulinemia is due to cold-induced precipitation of the cryoglobulins and resultant hyperviscosity and sludging. Clinical features include Raynaud's phenomenon, digital ischemia, livedo reticularis, and central nervous system involvement. This type of cryoglobulin almost always occurs in patients with lymphoproliferative disorders and treatment is directed at the underlying disorder.

Type II and type III cryoglobulins are found in states of chronic infection, such as hepatitis $\mathrm{C}$, and connective tissue diseases, such as Sjögrens and systemic lupus erythematosus. Tissue and organ damage is caused by deposition of immune complexes in blood vessels with resulting immune-complex mediated inflammation. The clinical syndrome seen in patients with circulating type II or III cryoglobulins was first described in 1966 as "Meltzer's triad" of purpura, arthalgias, and weakness
$[2,3]$. In addition, peripheral neuropathy and membranoproliferative glomerulonephritis are also a part of this syndrome, also referred to mixed cryoglobulinemic vasculitis. The majority of cases of cryoglobulinemic vasculitis are due to type II and III cryoglobulins, and an estimated $60-90 \%$ of mixed cryoglobulinemic vasculitis due to type II and III cryoglobulins is caused by chronic hepatitis $\mathrm{C}$ virus (HCV) infection [4-7]. This review will focus mainly on HCV-related cryoglobulinemic vasculitis.

The general approach to treatment of mixed cryoglobulinemic vasculitis is usually three-pronged:

1. Treatment of the underlying cause, such as with antiviral therapy in $\mathrm{HCV}$-associated disease

2. Target circulating B cells with rituximab (or other agents) to decrease $\mathrm{B}$ cell production of cryoglobulins in moderate to severe disease

3. Addition of plasmapheresis in combination with intensive immunosuppression (e.g., high-dose glucocorticoids and cyclophosphamide) to remove and prevent production of circulating cryoglobulins in cases of severe, life-threatening disease

\section{Treatment}

\section{Antiviral therapy}

\section{Treatment of hepatitis C infection}

Interferon-containing regimens

For the past decade, treatment of chronic HCV as well as HCV-

related cryoglobulinemic vasculitis has traditionally been the combination of PEGylated interferon (PEG-IFN) alpha and ribavirin. In one study [8], nine patients with HCV-associated cryoglobulinemic vasculitis were treated with PEG-IFN alpha-2b (1.5 $\mu \mathrm{g} / \mathrm{kg} /$ week) subcutaneously plus oral ribavirin (800-1200 mg/day) for at least 6 months. Patients were treated for a mean of $13.5 \pm 2.8$ months; $78 \%$ had a sustained virologic response and achieved a complete clinical response in their manifestations of cryoglobulinemic vasculitis. In a retrospective analysis of 10 patients with cryoglobulinemic glomerulonephritis treated with PEG-IFN alpha plus ribavirin, $40 \%$ achieved a sustained virologic response and $30 \%$ had a significant improvement in glomerulonephritis [9]. In a long-term follow up study of 40 patients treated with PEG-IFN alpha plus ribavirin (an average of 40 months after discontinuation of therapy), $67.5 \%$ had a sustained clinical response, $62.5 \%$ had a sustained virologic response, and $57.5 \%$ had a sustained immunologic response independent of HCV 
genotype and viral load. Absence of renal insufficiency was associated with a complete clinical response [10]. While PEG-IFN-based regimens show clinical efficacy, their inability to induce a complete clinical and virologic response in many patients indicates that other therapeutic options are needed.

More recently, treatment options have expanded with the addition of first-generation protease inhibitors (boceprevir or telaprevir). The efficacy of these regimens in the treatment of HCV-associated cryoglobulinemic vasculitis has been evaluated in several studies. The most relevant to clinical practice is an open-label, prospective, cohort study of 30 patients with HCV-related cryoglobulinemic vasculitis treated with PEG-IFN alpha and ribavirin for 48 weeks, and with either telaprevir (750 mg three times daily for 12 weeks) or boceprevir ( $800 \mathrm{mg}$ three times daily for 44 weeks) [11]. Complete clinical response, defined as resolution of purpura, arthralgia, weakness, and improvement of neuropathy and renal involvement, was achieved in $66.7 \%$ of patients and a partial clinical response, defined as achievement of three of the five complete response criteria, in $33.3 \%$ at week 72 . Serious adverse events occurred in $46.6 \%$ of patients, with high rates of infection, fatigue, cytopenias, and depression. Significant cytopenia was observed in $40 \%$ of patients, with $93 \%$ receiving erythropoietin, $46.6 \%$ requiring a blood transfusion, and $6.6 \%$ receiving a granulocyte stimulating agent.

Contraindications to use of interferon-containing regimens include advanced age, decompensated cirrhosis, major uncontrolled depression, significant coronary artery disease, and untreated thyroid disease [12]. Dosing of ribavirin depends on body weight and renal function. Combination therapy with ribavirin and PEG-IFN is contraindicated in patients with $\mathrm{CrCl}<50 \mathrm{ml} /$ minute, as well as in women who are pregnant and in men with pregnant partners $[13 \bullet \bullet]$.

Hepatitis $\mathrm{C}$ treatment has changed dramatically in the last several years with the advent of new direct acting antivirals that allow for the treatment of HCV without interferon. Current interferon-free regimens include ledipasvir-sofosbuvir without ribavirin, elbasvir-grazoprevir with or without ribavirin, ombitasvir-paritaprevir-ritonavir plus dasabuvir with or without ribavirin, simeprevir plus sofosbuvir with or without ribavirin, and daclatasvir plus sofosbuvir. The choice of regimen depends on HCV genotype, presence of cirrhosis, treatment history, and renal function, and other

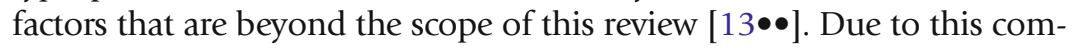
plexity, collaboration with a hepatologist is recommended. All oral interferon-free nucleotide polymerase inhibitors (with or without ribavirin) have shown high rates of sustained virologic responses (over $95 \%$ especially in HCV genotype 1 patients) in chronic HCV infections without many of the side effects of the interferon-containing regimens [14-16]. This favorable response profile has led to interest in the study of these regimens in patients with HCV-related cryoglobulinemic vasculitis.

The VASCUVALDIC study was an open-label prospective study evaluating the safety and efficacy of sofosbuvir, a nucleotide polymerase inhibitor, 
and ribavirin in 24 patients with active HCV-associated cryoglobulinemic vasculitis $[17 \bullet \bullet]$. Patients were treated with sofosbuvir $400 \mathrm{mg}$ daily plus ribavirin (200-1400 mg/day orally) for 24 weeks. Seven out of the 24 patients were treated with immunosuppressive or immunomodulatory therapy due to kidney involvement, severe peripheral neuropathy, or both. Thirteen patients were virologic non-responders to a previous antiviral therapy. At week $24,87.5 \%$ of patients achieved a complete clinical response, defined as improvement in all of the affected organs involved at baseline and the absence of clinical relapse, and the remaining $12.5 \%$ reached a partial clinical response, defined as an improvement in some but not all organs involved at baseline. Of the 24 patients, $74 \%$ had a sustained virologic response at 12 weeks after treatment.

Adverse events in this study included three (12.5\%) subjects who developed grade 3 and 4 anemia. Thirteen patients (54\%) received erythropoietin and three $(12.5 \%)$ received blood transfusions. However, the incidence of anemia in this study was much lower compared to the reported rates with interferon-containing regimens.

Some experts recommend delaying the initiation of antiviral therapy in patients with severe manifestations of cryoglobulinemic vasculitis for one to four months after initiation of immunosuppression [18]. This approach was investigated in a study using rituximab and PEG-IFN and ribavarin (discussed below) [19•]. The primary rationale for this approach comes from experience with interferon-containing regimens, which have been associated with flares of disease and immune-mediated events attributed to interferon use. Another rationale is that significant renal impairment from glomerulonephritis may preclude the use of direct acting antivirals and that treatment with immunosuppression may lead to an improvement in renal function that then allows for their use. More study is needed to determine optimal timing for the initiation of antiviral medications.

Although there is a lack of robust data to support the use of interferonfree and ribavirin-free antiviral regimens in the treatment of patients with HCV-associated cryoglobulinemic vasculitis, extrapolation from the results of treatment of chronic HCV would argue for the use of these agents as firstline antiviral therapy for HCV-related cryoglobulinemic vasculitis.

\section{Treatment of hepatitis B infection}

While cryoglobulins and cryoglobulinemic vasculitis are detected in less than $5 \%$ of patients with chronic hepatitis B virus (HBV) infection [20], cryoglobulinemic vasculitis due to HBV can be organ and life-threatening. Similar to HCV-related cryoglobulinemic vasculitis discussed above, use of antiviral therapy is generally considered first-line therapy. Case reports support the use of nucleoside/nucleotide analogs such as lamivudine and entecavir in the treatment of HBV-associated cryoglobulinemic vasculitis [21, 22]. Entecavir is generally favored because of a lower risk of resistance and nephrotoxicity. Generally, antiviral therapy should be started as soon as possible, especially if immunosuppression is planned, given the risk of increased viral replication and worsening liver disease. Unlike with interferon, the initiation of entecavir and other antivirals directed at hepatitis B is not associated with an increased risk of immune-mediated events or flare of cryoglobulinemic vasculitis. 


\section{Glucocorticoids}

The use of glucocorticoids for the treatment of HCV-associated cryoglobulinemic vasculitis is controversial and practice patterns vary significantly. There are no randomized controlled trials evaluating the safety and efficacy of glucocorticoids in HCV-associated cryoglobulinemic vasculitis. A Cochran review from 2004 analyzed eight randomized clinical trials that used glucocorticoids for the treatment of hepatitis $\mathrm{C}$ with or without associated autoimmune disorders. There was insufficient evidence to confirm either harm or benefit of glucocorticoids in this setting [23].

In patients with organ- or life-threatening manifestations of HCVassociated cryoglobulinemic vasculitis, a short course of high-dose pulse glucocorticoids is often used in combination with other immunosuppressive therapies. This practice is based on data from small uncontrolled studies [24]. The two randomized controlled trials of rituximab in HCVassociated glomerulonephritis allowed concomitant glucocorticoid use and dosing at the discretion of the investigator. There was no difference in outcomes detected in patients treated with glucocorticoids. However, the patients with more severe disease almost uniformly received them, and thus it is difficult to draw conclusions about potential risks and benefits

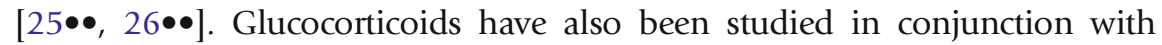
interferon-alpha in a randomized controlled trial of interferon-alpha with and without moderate dose prednisolone $(16 \mathrm{mg} /$ day $)$ for one year in patients with type II mixed cryoglobulinemic vasculitis. There was a more rapid time to complete response in the group treated with combination therapy compared with monotherapy with IFN-alpha and a higher risk of relapse in the group treated with IFN-alpha monotherapy compared with combination therapy [27]. However, this approach is less relevant to current clinical practice given advances in antiviral treatment.

In a small cohort study of five patients with HCV-associated glomerulonephritis treated with rituximab, steroids were not used. Patients had a rapid and sustained response, although relapse was common. Only one patient was treated with low-dose steroids 18 months after rituximab for persistent arthralgias [28].

In light of the lack of clear evidence of benefit of glucocorticoids and significant risk of harm, especially with long-term use, we recommend a cautious approach to their use. In general, we support the use of high-dose pulse glucocorticoids in the setting of severe disease with prompt initiation of antivirals and/or steroid-sparing immunosuppressant medications to allow a rapid steroid taper.

\section{Rituximab}

Cryoglobulinemic vasculitis is characterized by the clonal expansion of B cells that produce IgM and $\operatorname{IgG}$ rheumatoid factor, leading to immune-complex formation, deposition in blood vessel walls, resultant inflammation, and tissue injury. Rituximab (RTX) is a chimeric monoclonal antibody targeted against CD20, a B-lymphocyte cell surface antigen expressed in all stages except the first 
(early pro-B cell) and last (plasma cell) stages of B cell development. The use of rituximab results in $\mathrm{CD} 20+\mathrm{B}$ cell depletion. RTX was hypothesized to be a potential therapy for cryoglobulinemic vasculitis since RTX may deplete the B cell population that contributes to cryoglobulin production.

Since first-line therapy for many patients with cryoglobulinemic vasculitis is antiviral therapy, RTX was studied in combination with PEG-IFN alpha and ribavirin. In a study by Dammacco et al., patients with HCV-related cryoglobulinemic vasculitis were randomized to receive either RTX $(375 \mathrm{mg} /$ $\mathrm{m}^{2}$ /week for 4 weeks) concurrently with PEG-IFN and ribavarin followed by one RTX infusion $\left(375 \mathrm{mg} / \mathrm{m}^{2}\right)$ at month 6 and $11(n=22)$ or the standard therapy of PEG-IFN and ribavirin without RTX $(n=15)$. At 12 months, $54 \%$ of the RTX group and $33 \%$ of the standard therapy group had a complete response $(p<0.05)$. Of those who responded, $83 \%$ in the RTX group and $40 \%$ in the standard therapy group maintained their response for up to 3 years $(p<0.01)$ [29]. In a prospective cohort study, 38 patients initially treated with rituximab $\left(375 \mathrm{mg} / \mathrm{m}^{2} /\right.$ week for 4 weeks), and then treated with PEG-IFN alpha weekly plus ribavirin daily for 48 weeks, were compared to 55 patients treated with PegIFN alpha/ribavirin [19•]. Patients in the rituximab treatment group had a shorter time to clinical remission ( $5.4 \pm 4$ vs. $8.4 \pm 4.7$ months, $p=0.004)$, and better renal response rates ( 80.9 vs. $40 \%, p=0.040$ ). These two studies indicate the efficacy of rituximab use with PEG-IFN/ribavarin therapy for the treatment of HCV-related cryoglobulinemic vasculitis. In addition, the results of the second study indicated that delaying antiviral therapy for 4 weeks was not associated with a worse outcome.

RTX has been studied in two randomized controlled clinical trials of the treatment of cryoglobulinemic vasculitis in patients with and without HCVassociated disease, and was shown to be superior when compared to conventional immunosuppressive therapies $[25 \bullet \bullet, 26 \bullet \bullet]$. Patients in these trials with $\mathrm{HCV}$-associated cryoglobulinemic vasculitis had either previously failed treat-

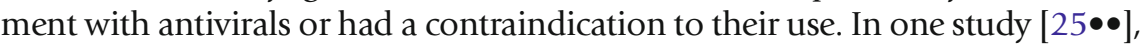
59 patients with cryoglobulinemic vasculitis and related skin ulcers, active glomerulonephritis, or refractory peripheral neuropathy were randomized receive RTX ( 1 gm IV 2 weeks apart, with a second course at relapse if needed) or other therapy (treated with either glucocorticoid; azathioprine or cyclophosphamide; or plasmapheresis). The primary endpoint, survival at 12 months, was higher in the RTX group (64.3 vs. $3.5 \%, p<0.0001$ ). The median duration of clinical response was 18 months in the RTX-treated group and patients were retreated at relapse with RTX. In the second study [26••], 24 patients with HCVassociated cryoglobulinemic vasculitis were randomized to treatment with either RTX ( $375 \mathrm{mg} / \mathrm{m}^{2} /$ week for 4 weeks) or best available therapy, which included azathioprine, glucocorticoids, and cyclophosphamide. The primary endpoint, remission at study month 6 (which was defined by a Birmingham Vasculitis Activity Score of 0 ), was reached in $83 \%$ of the RTX group compared with $8.3 \%$ of the control group ( $p<0.001)$.

There is little data on the use of rituximab in non-HCV-cryoglobulinemic vasculitis. The CryoVas survey was a retrospective survey of physicians from French hospitals and included 242 patients with non-infectious mixed cryoglobulinemic vasculitis. This group included patients with connective tissue diseases $(n=73)$, idiopathic or essential disease $(n=117)$, and hematologic malignancies $(n=52)$. First-line treatment with rituximab plus glucocorticoids 
resulted in a complete clinical response in $64 \%$ of patients compared with $44 \%$ in patients treated with glucocorticoids alone and $62 \%$ in patients treated with alkylating agents and glucocorticoids [30]. In another prospective study, the safety and efficacy of treatment with rituximab in twenty three patients with non-HCV mixed cryoglobulinemic vasculitis from the French Autoimmunity and Rituximab Registry (AIR) was evaluated. Follow up data was available in twenty patients. Seventeen $(85 \%)$ patients had a complete clinical response and mean prednisone dose decreased from $17.5 \mathrm{mg} /$ day to $5 \mathrm{mg} /$ day $(p=0.0005)$ at 6 months after treatment with rituximab. Severe infections occurred in six of twenty three (26\%) of patients, which was similar to what was reported in the CryoVas survey [31]. These results suggest that rituximab is effective in the treatment of non-HCV mixed cryoglobulinemic vasculitis, but close monitoring for infectious complications is important.

Side effects from RTX include infusion reactions, infections, and hypogammaglobulinemia. Infectious risks do not seem to be lower with RTX

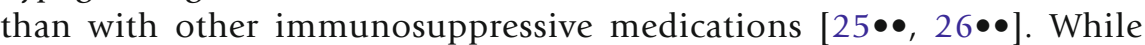
Pneumocystis prophylaxis is frequently considered for patients with ANCAassociated vasculitis treated with rituximab, it is not routinely used for cryoglobulinemic vasculitis patients treated with rituximab. Hypogammaglobulinemia developed in only one patient in the clinical trials mentioned above who had previously been treated with high-dose corticoste-

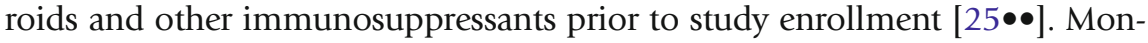
itoring serum IgG levels at baseline and then periodically (every 3 to 6 months) is a reasonable approach, especially in patients who have previously been immunosuppressed and/or have received multiple courses of RTX. Treatment with RTX does not appear to increase liver injury in patients with HCV, with no increases in HCV plasma viremia [26••] or in hepatic transaminase levels reported $[25 \bullet \bullet, 26 \bullet \bullet]$. Serum sickness after treatment with RTX has been reported [32] but was not seen in the randomized clinical trials. Progressive multifocal leukoencephalopathy, a rare neurologic disease caused by reactivation of the JC virus, has been associated with use of RTX [33], although no cases have been reported in patients with cryoglobulinemic vasculitis treated with RTX at this time.

Contraindications to RTX use include active (non-HCV) infection. Current active or past hepatitis B infection is a particular concern, since rituximab use can worsen or reactivate hepatitis B infection, leading to fulminant liver failure.

\section{Cyclophosphamide}

While the role of cyclophosphamide (CYC) as a first-line therapeutic agent in ANCA-associated vasculitis is well-accepted [34], the role of CYC in the treatment of HCV-related cryoglobulinemic vasculitis is less clear. The use of cyclophosphamide in cryoglobulinemic vasculitis has not been rigorously studied in clinical trials. CYC is generally considered when patients fail to respond to antiviral and/or rituximab therapy, or when these therapies are not tolerated [35]. CYC is also used in conjunction with other therapies such as plasma exchange to suppress the formation of cryoglobulins. CYC use for HCVrelated cryoglobulinemic vasculitis is limited, in part, because CYC may induce liver dysfunction and increase viral replication. 
The treatment of non-HCV-related cryoglobulinemic vasculitis is not well defined. The European League Against Rheumatism (EULAR) recommends that non-viral cryoglobulinemic vasculitis be treated with immunosuppressive agents [36], and CYC may have a more prominent role in the treatment of this entity. However, other agents, including rituximab [37], may be efficacious as well.

Given the lack of dedicated studies of CYC use in cryoglobulinemic vasculitis, the CYC regimen used to treat ANCA-associated vasculitis is commonly applied to this disease. Usual initial doses are $2 \mathrm{mg} / \mathrm{kg} / \mathrm{day}$ by mouth, adjusted for age and renal insufficiency. Treatment courses are generally limited to 3 months (no more than 6 months), after which CYC is stopped and a less toxic medication is used, such as azathioprine or potentially mycophenolate mofetil (methotrexate is generally avoided

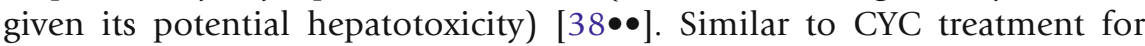
other autoimmune diseases, toxicities from CYC use include increased infectious risk, hemorrhagic cystitis, leukopenia, and heptatotoxicity. Patients taking CYC should maintain adequate hydration $(2 \mathrm{~L}$ of liquids per day are recommended) to decrease the risk of bladder toxicity and also receive prophylaxis against Pneumocystis jirovecii pneumonia. Dosedependent risks include malignancy and gonadal failure. Contraindications to CYC use include pregnancy due to its teratogenic nature, active infection, non-immune-mediated leukopenia, and a prior history of hemorrhagic cystitis.

In therapeutic plasma exchange, blood is removed from the body, separated into cells and plasma, with the cells returned to the body and the plasma discarded. Removal of the plasma removes large molecular weight proteins such as antibodies and cryoglobulins from the blood. Plasma exchange is not commonly used for the treatment of cryoglobulinemic vasculitis. Based on multiple case reports and case series, it can be considered first-line therapy for severe life- or organthreatening manifestations of cryoglobulinemic vasculitis, such as alveolar hemorrhage, acute gastrointestinal involvement, and rapidly progressive glomerulonephritis [39]. In these situations, it can be used as a bridge to treatment of the underlying disease or initiation of immunosuppressive agents such as rituximab. Plasma exchange has also been used to treat refractory cutaneous involvement (e.g., skin ulcerations not responsive to systemic immunosuppression). Since plasma exchange does not prevent the new formation of cryoglobulins, this therapeutic modality needs to be used in conjunction with other immunosuppressive agents such as glucocorticoids, cyclophosphamide, or rituximab to suppress additional cryoglobulin production from clonal B cells.

The case reports and cases series supporting the use of plasma exchange employed widely different plasma exchange protocols [18]. In general, $1-1.5 \times$ total plasma volume is exchanged, with a $5 \%$ albumin used as the replacement solution [39]. Plasma has also been 
used as the replacement solution, and is recommended if the patient has an increased risk of bleeding or will be undergoing an invasive procedure (e.g., surgery or organ biopsy). The number and schedule of treatments vary, ranging from 3 sessions/week for 2-3 weeks to 10-14 daily sessions. Warming the replacement fluid as well as the draw and return lines are recommended to prevent cryoglobulin precipitation within the extracorporeal circuit.

Since the cryocrit (the measured concentration of cryoglobulin in the serum) does not correlate well with disease severity or therapeutic efficacy, the decision to initiate or continue plasma exchange should be based on the patient's disease severity and therapeutic response. Clinical disease activity should begin to improve after the initiation of plasma exchange (e.g., improvement in serum creatinine if initiated for glomerulonephritis, or improvement in skin ulceration or purpura if initiated for cutaneous involvement). The one exception is neuropathy, which is unlikely to improve rapidly.

If albumin is used as the replacement fluid, levels of blood clotting factors may transiently decrease but generally correct within 24 hours. Patients undergoing surgical procedures should have their prothrombin and activated partial thromboplastin times assessed. Other risks include fluid overload or under-replacement, and anaphylactic or allergic reactions to the plasma transfusion.

Interleukin-2 (IL-2) is a cytokine that mediates the differentiation and survival of T-regulatory (Tregs) cells as well as effector $\mathrm{T}$ cell responses, and is approved as adjunct therapy for the treatment of renal cell carcinoma. Treg populations have been noted to expand after treatment with IL-2 therapy. Patients with HCV-related mixed cryoglobulinemia have decreased Treg counts. Increased Treg counts are associated with clearance of the virus and cure of vasculitis [19•, 40]. Thus, IL-2 has been proposed as a potential therapeutic option for patients with HCVrelated mixed cryoglobulinemia.

The VASCU-IL2 study [41] was an open-label phase I-IIA study of ten patients with HCV-related vasculitis refractory to antiviral therapy and/or rituximab and not receiving glucocorticoids or other immunosuppressants. These patients received 4 courses of low-dose IL-2 subcutaneously: 1.5 million IU/day $\times 5$ days, followed by three 5 -day courses of 3 million IU/day at weeks 3, 6, and 9. Eight of the ten patients noted clinical improvement in purpura, arthralgias, and renal function (1 patient) by the second course of therapy; the two patients that did not improve had neuropathy as their sole clinical manifestation. The only severe adverse event noted was one patient who was hospitalized with a dental abscess. The proportion of Tregs increased from a mean baseline value of $3.6 \%$ to a median peak value of $14 \%$. HCV viral load, C4, and cryoglobulin levels were not significantly affected. This study indicates that low-dose IL-2 use can be therapeutic for mixed cryoglobulinemia due to HCV, and indicates a new possible avenue of therapy-modulating the immune response to the virus instead of targeting the virus itself. 


\section{Conclusions}

In summary, treatment of HCV-related cryoglobulinemic vasculitis has made significant advances due to the increasingly effective antiviral therapies directed against HCV. Antiviral therapies may be curative for HCV-associated cryoglobulinemic vasculitis if HCV eradication is achieved in the patient. Immunosuppressive agents such as rituximab continue to have an important adjunctive role, especially for patients with organ- or life-threatening disease, and may be initiated prior to antiviral therapy. Plasmapheresis can also be used as a bridge to more definitive therapies during flares of severe disease. While some studies support the use of rituximab for non-HCV-related cryoglobulinemic vasculitis, the optimal treatment regimen for this type of vasculitis remains unclear, and would benefit from additional study.

\section{Compliance with Ethical Standards}

\section{Conflicts of Interest}

Sarah Goglin and Sharon Chung declare that they have no conflicts of interest.

Human and Animal Rights and Informed Consent

This article does not contain any studies with human or animal subjects performed by any of the authors.

\section{References and Recommended Reading}

Papers of particular interest, published recently, have been highlighted as:

- Of importance

$\bullet \quad$ Of major importance

1. Brouet JC, Clauvel JP, Danon F, Klein M, Seligmann M. Biologic and clinical significance of cryoglobulins. A report of 86 cases. Am J Med. 1974;57:775-88.

2. Meltzer M, Franklin EC. Cryoglobulinemia-a study of twenty-nine patients. I. IgG and IgM cryoglobulins and factors affecting cryoprecipitability. Am J Med. 1966;40:828-36.

3. Meltzer M, Franklin EC, Elias K, McCluskey RT, Cooper N. Cryoglobulinemia-a clinical and laboratory study. II. Cryoglobulins with rheumatoid factor activity. Am J Med. 1966;40:837-56.

4. Cacoub P, Poynard T, Ghillani P, et al. Extrahepatic manifestations of chronic hepatitis C. MULTIVIRC Group. Multidepartment Virus C. Arthritis Rheum. 1999;42:2204-12.

5. Trejo O, Ramos-Casals M, Garcia-Carrasco M, et al. Cryoglobulinemia: study of etiologic factors and clinical and immunologic features in 443 patients from a single center. Medicine. 2001;80:252-62.

6. Saadoun D, Sellam J, Ghillani-Dalbin P, Crecel R, Piette JC, Cacoub P. Increased risks of lymphoma and death among patients with non-hepatitis $\mathrm{C}$ virusrelated mixed cryoglobulinemia. Arch Intern Med. 2006;166:2101-8.

7. Ferri C, Zignego AL, Pileri SA. Cryoglobulins. J Clin Pathol. 2002;55:4-13.

8. Cacoub P, Saadoun D, Limal N, Sene D, Lidove O, Piette J-C. PEGylated interferon alfa-2b and ribavirin treatment in patients with hepatitis $\mathrm{C}$ virus-related systemic vasculitis. Arthritis Rheum. 2005;52:911-5.

9. Mazzaro C, Monti G, Saccardo F, et al. Efficacy and safety of peginterferon alfa-2b plus ribavirin for $\mathrm{HCV}$ positive mixed cryoglobulinemia: a multicentre openlabel study. Clin Exp Rheumatol. 2011;29:933-41. 
10. Saadoun D, Resche-Rigon M, Thibault V, Piette J-C, Cacoub P. Antiviral therapy for hepatitis C virusassociated mixed cryoglobulinemia vasculitis: a longterm follow-up study. Arthritis Rheum. 2006;54:3696706.

11. Saadoun D, Resche Rigon M, Pol S, et al. PegIFNalpha/ ribavirin/protease inhibitor combination in severe hepatitis $C$ virus-associated mixed cryoglobulinemia vasculitis. J Hepatol. 2015;62:24-30.

12. Ghany MG, Strader DB, Thomas DL, Seeff LB, American Association for the Study of Liver D. Diagnosis, management, and treatment of hepatitis C: an update. Hepatology. 2009;49:1335-74.

13.• Panel AIHG. Hepatitis C guidance: AASLD-IDSA recommendations for testing, managing, and treating adults infected with hepatitis $\mathrm{C}$ virus. Hepatology. 2015;62:932-54.

These are the consensus guidelines with current ad specific recommendations for treatment of adults with hepatitis $\mathrm{C}$ virus.

14. Gane EJ, Stedman CA, Hyland RH, et al. Nucleotide polymerase inhibitor sofosbuvir plus ribavirin for hepatitis C. N Engl J Med. 2013;368:34-44.

15. Lawitz E, Mangia A, Wyles D, et al. Sofosbuvir for previously untreated chronic hepatitis $\mathrm{C}$ infection. $\mathrm{N}$ Engl J Med. 2013;368:1878-87.

16. Lawitz E, Poordad FF, Pang PS, et al. Sofosbuvir and ledipasvir fixed-dose combination with and without ribavirin in treatment-naive and previously treated patients with genotype 1 hepatitis $\mathrm{C}$ virus infection (LONESTAR): an open-label, randomised, phase 2 trial.

17.• Saadoun D, Thibault V, Si Ahmed SN, et al. Sofosbuvir plus ribavirin for hepatitis $\mathrm{C}$ virus-associated cryoglobulinaemia vasculitis: VASCUVALDIC study. Ann Rheum Dis. 2015.

This study provides the only data on the use of sofosbuvir plus ribavirin in the treatment of $\mathrm{HCV}$-associated vasculitis. It is the only study thus far to evaluate one of the new direct-acting antiviral non-interfon regimens in this disease.

18. Pietrogrande M, De Vita S, Zignego AL, et al. Recommendations for the management of mixed cryoglobulinemia syndrome in hepatitis $\mathrm{C}$ virusinfected patients. Autoimmun Rev. 2011;10:444-54.

19. Saadoun D, Resche Rigon M, Sene D, et al. Rituximab plus Peg-interferon-alpha/ribavirin compared with Peg-interferon-alpha/ribavirin in hepatitis C-related mixed cryoglobulinemia. Blood. 2010;116:326-34.

quiz 504-5. This trial provides support for the use of rituximab as adjunctive treatment to PEG-IFN/ribavirin in the treatment of HCV-associated cryoglobulinemic vasculitis.

20. Cacoub P, Saadoun D, Bourlière $M$, et al. Hepatitis B virus genotypes and extrahepatic manifestations. J Hepatol. 2005;43:764-70.

21. Enomoto M, Nakanishi T, Ishii M, Tamori A, Kawada N. Entecavir to treat hepatitis B-associated cryoglobulinemic vasculitis. Ann Intern Med. 2008;149:912-3.
22. Viganò $M$, Martin $P$, Cappelletti M, Fabrizi F. HBVassociated cryoglobulinemic vasculitis: remission after antiviral therapy with entecavir. Kidney Blood Press Res. 2014;39:65-73.

23. Brok J, Mellerup Martin T, Krogsgaard K, Gluud C. Glucocorticosteroids for viral hepatitis C. Cochrane Database of Systematic Reviews: John Wiley \& Sons, Ltd; 2004.

24. De Vecchi A, Montagnino G, Pozzi C, Tarantino A, Locatelli F, Ponticelli C. Intravenous methylprednisolone pulse therapy in essential mixed cryoglobulinemia nephropathy. Clin Nephrol. 1983;19:221-7.

$25 . \bullet$ De Vita S, Quartuccio L, Isola M, et al. A randomized controlled trial of rituximab for the treatment of severe cryoglobulinemic vasculitis. Arthritis Rheum. 2012;64:843-53.

This randomized controlled trial provides the basis for the use of rituximab in the treatment of both HCV-associated and non$\mathrm{HCV}$-associated cryoglobulinemic vasculitis.

26.• Sneller MC, Hu Z, Langford CA. A randomized controlled trial of rituximab following failure of antiviral therapy for hepatitis $\mathrm{C}$ virus-associated cryoglobulinemic vasculitis. Arthritis Rheum. 2012;64:835-42.

This randomized controlled trial provides the basis for the use of rituximab in the treatment of HCV-associated cryoglobulinemic vasculitis in patients who have failed previous interferon-based treatment of HCV.

27. Dammacco F, Sansonno D, Han JH, et al. Natural interferon-alpha versus its combination with 6-methylprednisolone in the therapy of type II mixed cryoglobulinemia: a long-term, randomized, controlled study. Blood. 1994;84:3336-43.

28. Quartuccio L, Soardo G, Romano G, et al. Rituximab treatment for glomerulonephritis in $\mathrm{HCV}$-associated mixed cryoglobulinaemia: efficacy and safety in the absence of steroids. Rheumatology. 2006;45:842-6.

29. Dammacco F, Tucci FA, Lauletta G, et al. Pegylated interferon- $\alpha$, ribavirin, and rituximab combined therapy of hepatitis $\mathrm{C}$ virus-related mixed cryoglobulinemia: a long-term study. Blood. 2010;116:343-53.

30. Terrier B, Krastinova E, Marie I, et al. Management of noninfectious mixed cryoglobulinemia vasculitis: data from 242 cases included in the CryoVas survey. Blood. 2012;119:5996-6004.

31. Terrier B, Launay D Fau - Kaplanski G, Kaplanski G Fau - Hot A, et al. Safety and efficacy of rituximab in nonviral cryoglobulinemia vasculitis: data from the French Autoimmunity and Rituximab registry.

32. Sene D, Ghillani-Dalbin P, Amoura Z, Musset L, Cacoub P. Rituximab may form a complex with IgMkappa mixed cryoglobulin and induce severe systemic reactions in patients with hepatitis $C$ virusinduced vasculitis. Arthritis Rheum. 2009;60:3848-55.

33. Molloy ES, Calabrese LH. Progressive multifocal leukoencephalopathy associated with 
immunosuppressive therapy in rheumatic diseases: evolving role of biologic therapies. Arthritis Rheum. 2012;64:3043-51.

34. Stone JH, Merkel PA, Spiera R, et al. Rituximab versus cyclophosphamide for ANCA-associated vasculitis. N Engl J Med. 2010;363:221-32.

35. Ferri C, Sebastiani M, Antonelli A, Colaci M, Manfredi A, Giuggioli D. Current treatment of hepatitis Cassociated rheumatic diseases. Arthritis Res Ther. 2012;14:215.

36. Mukhtyar C, Guillevin L, Cid MC, et al. EULAR recommendations for the management of primary small and medium vessel vasculitis. Ann Rheum Dis. 2009;68:310-7.

37. Terrier B, Launay D, Kaplanski G, et al. Safety and efficacy of rituximab in nonviral cryoglobulinemia vasculitis: data from the French Autoimmunity and Rituximab registry. Arthritis Care Res. 2010;62:178795.
38.• De Vita S, Quartuccio L, Isola M, et al. A randomized controlled trial of rituximab for the treatment of severe cryoglobulinemic vasculitis. Arthritis Rheum. 2012;64:843-53.

This randomized controlled trial provides the basis for the use of rituximab in the treatment of cryoglobulinemic vasculitis.

39. Szczepiorkowski ZM, Winters JL, Bandarenko N, et al. Guidelines on the use of therapeutic apheresis in clinical practice-evidence-based approach from the Apheresis Applications Committee of the American Society for Apheresis. J Clin Apher. 2010;25:83-177.

40. Landau DA, Rosenzwajg M, Saadoun D, TrebedenNegre H, Klatzmann D, Cacoub P. Correlation of clinical and virologic responses to antiviral treatment and regulatory $\mathrm{T}$ cell evolution in patients with hepatitis $\mathrm{C}$ virus-induced mixed cryoglobulinemia vasculitis. Arthritis Rheum. 2008;58:2897-907.

41. Saadoun D, Rosenzwajg M, Joly F, et al. Regulatory Tcell responses to low-dose interleukin-2 in HCVinduced vasculitis. N Engl J Med. 2011;365:2067-77. 